\title{
Lysyl oxidase enzymes mediate TGF- $\beta 1$-induced fibrotic phenotypes in human skin-like tissues
}

\author{
Mengqi Huang ${ }^{1,2,6} \cdot$ Zhiyi Liu $^{3,7} \cdot$ Lauren Baugh $^{3} \cdot$ Jason DeFuria ${ }^{1} \cdot$ Anna Maione ${ }^{1}$ Avi Smith ${ }^{1}$ Olga Kashpur ${ }^{1}$. \\ Lauren D. Black III $\mathbb{1}^{3,4} \cdot$ Irene Georgakoudi ${ }^{3} \cdot$ Michael L. Whitfield ${ }^{2,5} \cdot$ Jonathan Garlick $^{1}$
}

Received: 30 May 2018 / Revised: 17 October 2018 / Accepted: 29 October 2018 / Published online: 19 December 2018

(c) United States \& Canadian Academy of Pathology 2018

\begin{abstract}
Cutaneous fibrosis is a common complication seen in mixed connective tissue diseases. It often occurs as a result of TGF- $\beta$ induced deposition of excessive amounts of collagen in the skin. Lysyl oxidases (LOXs), a family of extracellular matrix (ECM)-modifying enzymes responsible for collagen cross-linking, are known to be increased in dermal fibroblasts from patients with fibrotic diseases, denoting a possible role of LOXs in fibrosis. To directly study this, we have developed two bioengineered, in vitro skin-like models: human skin equivalents (hSEs), and self-assembled stromal tissues (SASs) that contain either normal or systemic sclerosis (SSc; scleroderma) patient-derived fibroblasts. These tissues provide an organlevel structure that could be combined with non-invasive, label-free, multiphoton microscopy (SHG/TPEF) to reveal alterations in the organization and cross-linking levels of collagen fibers during the development of cutaneous fibrosis, which demonstrated increased stromal rigidity and activation of dermal fibroblasts in response to TGF- $\beta 1$. Specifically, inhibition of specific LOXs isoforms, LOX and LOXLA, in foreskin fibroblasts (HFFs) resulted in antagonistic effects on TGF- $\beta 1$ induced fibrogenic hallmarks in both hSEs and SASs. In addition, a translational relevance of these models was seen as similar antifibrogenic phenotypes were achieved upon knocking down LOXL4 in tissues containing SSc patient-deriveddermal fibroblasts (SScDFs). These findings point to a pivotal role of LOXs in TGF- $\beta 1$-induced cutaneous fibrosis through impaired ECM homeostasis in skin-like tissues, and show the value of these tissue platforms in accelerating the discovery of antifibrosis therapeutics.
\end{abstract}

\section{Introduction}

Skin fibrosis is linked to excessive dermal deposition of collagenous and non-collagenous extracellular matrix (ECM) components as a consequence of aberrant

Electronic supplementary material The online version of this article (https://doi.org/10.1038/s41374-018-0159-8) contains supplementary material, which is available to authorized users.

Jonathan Garlick

jonathan.garlick@tufts.edu

1 Department of Diagnostic Sciences, Tufts University School of Dental Medicine, Boston, MA, USA

2 Department of Molecular and Systems Biology, Geisel School of Medicine at Dartmouth, Hanover, NH, USA

3 Department of Biomedical Engineering, Tufts University, Medford, MA, USA

4 Cellular, Molecular, and Developmental Biology Program, Sackler production and altered remodeling by fibroblasts [1, 2]. Pathologic hallmarks of dermal fibrosis include an increased number of $\alpha \mathrm{SMA}$-expressing myofibroblasts and the excess production and altered remodeling of collagenous ECM, leading to elevated tissue contraction and stiffness [3,4]. As a classical effector molecule of fibrogenesis, transforming growth factor $\beta$ (TGF- $\beta$ ) binds to its cognate receptor on fibroblasts, promoting fibroblast-myofibroblast transdifferentiation and contributing significantly to tissue fibrosis [5]. TGF- $\beta 1$ is the

School for Graduate Biomedical Sciences, Tufts University School of Medicine, Boston, MA, USA

5 Department of Biomedical Data Science, Geisel School of Medicine at Dartmouth, Lebanon, NH, USA

6 Department of Stomatology, Tongji Hospital, Tongji Medical College, Huazhong University of Science and Technology, Wuhan, China

7 State Key Laboratory of Modern Optical Instrumentation, College of Optical Science and Engineering, Zhejiang University, Hangzhou, China 
isoform most commonly associated with skin fibrosis, as elevated levels of activated TGF- $\beta 1$ are found in the systemic circulation of patients with fibrotic disease and TGF- $\beta 1$ levels in skin lesions are correlated with the degree of skin fibrosis [6]. Additional pro-fibrotic mechanisms that could account for ECM alterations upon TGF- $\beta 1$ stimulation are largely unknown.

One possible mechanism for altered ECM remodeling is through collagen cross-linking. The lysyl oxidase family of enzymes (LOXs) contains five genes, LOX and LOX-like (LOXL1-4), that function to covalently cross-link collagens via deamination of lysine residues [7]. This cross-linking reaction provides mechanical strength to the ECM thus making it more resistant to degradation [8]. It was previously shown that LOXs-mediated cross-linking might be a critical process in TGF- $\beta$ signaling, inducing fibrotic phenotypes by modifying ECM organization and tissue stiffness [9]. This study further investigates the role of LOXs-mediated collagen cross-linking to understand how TGF- $\beta$ regulates cutaneous fibrogenesis.

Previous researchers have demonstrated that human skin equivalents (hSEs) mimic their in vivo counterparts as they comprise both epithelium and underlying connective tissues $[10,11]$. In addition to hSEs, we developed a dermal model of self-assembled stromal tissues (SASs), in which fibroblasts secrete and organize ECM similar to granulation tissue, providing insights into de novo ECM deposition and remodeling [12]. Recapitulating pro-fibrotic phenotypes in two distinct 3D skin-like tissue systems has allowed us to identify changes in ECM organization by using noninvasive imaging, namely second-harmonic generation and two-photon excited fluorescence (SHG/TPEF) microscopy. We previously validated that the TPEF signal is strongly correlated to liquid chromatography-mass spectrometry (LC/MS) measurements of LOXs-mediated crosslinks, which is a traditional method to directly analyze collagen cross-linking [13]. However, sample preparation for LC/MS requires hydrolysis, which limits the detection of spatial heterogeneities in the cross-linking of small-scale samples. In this study, SHG/TPEF was used to compare collagen production and cross-linking with tissue stiffness measurements recorded by atomic force microscopy (AFM). We found that these 3D tissue models mimic key pro-fibrogenic features in the skin by revealing that ECM composition and organization were correlated with structural and mechanical properties of connective tissues. Our results showed that inhibition of LOXs blocked collagen cross-linking and resulted in dramatic alterations in ECM composition and structure, revealing that LOXs are critical for pro-fibrotic phenotypes. This suggested that the 3D tissue platforms and analytical image tools can be used to accelerate the discovery and test of antifibrotic therapies by enabling the screening of therapeutic compounds.

\section{Materials and methods}

\section{Cell culture}

Using a protocol approved by the Tufts Medical Center Institutional Review Board (IRB), a 4-mm in diameter and 3-mm depth skin biopsy was performed on the forearm of a de-identified, systemic sclerosis (SSc; scleroderma) patient and an age-, gender-matched health control. Dermal fibroblasts were isolated as previously described [14]. Briefly, the skin biopsy was incubated overnight at $4{ }^{\circ} \mathrm{C}$ in dispase (Invitrogen, CA) to remove the epidermis. The dermal tissues was minced and incubated in collagenase (Invitrogen, CA)/hyoluronidase (Sigma-Aldrich, MO)/DMEM-F12 (Invitrogen, $\mathrm{CA}$ ) for $1 \mathrm{~h}$ at $37^{\circ} \mathrm{C}$ with constant stirring. Red blood cell lysis buffer (Sigma-Aldrich, MO) was added for $2 \mathrm{~min}$, and the remaining cells and tissues were collected by centrifugation. Human foreskin fibroblasts (HFFs) and SSc dermal fibroblasts (SScDFs) were cultured in fibroblast growth medium containing $1 \mathrm{~g} / \mathrm{L}$ glucose DMEM (Invitrogen, CA), 10\% FBS (HyClone, UT), $0.19 \mathrm{~g} / \mathrm{mL}$ HEPES (Sigma-Aldrich, MO), and 100U Pen/Strep (Invitrogen, CA) at $37^{\circ} \mathrm{C}$ in a $5 \% \mathrm{CO}_{2}$ humidified atmosphere. At confluence, experiments were conducted on passages between 6 to 8 .

\section{D constructs}

hSEs were constructed in transwell inserts as previously described [8]. Briefly, the fibroblasts contracted and remodeled bovine collagen matrices for 7 days to generate human dermal equivalents (hDEs). Then, $5 \times 10^{5}$ keratinocytes isolated from human neonatal foreskin were seeded onto the plateau of collagen to make hSEs. Both hDEs and hSEs were submerged in epidermal growth medium $(0.3 \%$ bovine calf serum, organogenesis) for 5 days, then lifted to a liquid-air interface for 4 days, followed by additional 1,2, $5 \mathrm{ng} / \mathrm{mL}$ TGF- $\beta 1$ (R\&D, MN) or $8 \mu$ M BAPN (Sigma, MO) treatment for 8 days. The stromal layer was isolated from the epithermal layer by submerging hSEs in $2.4 \mathrm{U} / \mathrm{mL}$ Dispase (Invitrogen, CA) for $10 \mathrm{~min}$. To make SASs, fibroblasts were seeded onto $0.1 \mu \mathrm{m}$ polyethylene terephthalate membranes (Millipore, MA) at a density of $1.6 \times$ $10^{5}$ cells/insert. Self-assembly medium [12] supplemented with $10 \mu \mathrm{g} / \mathrm{mL}$ ascorbic acid (Sigma, MO) was changed twice every week for 2 weeks followed by $2 \mathrm{ng} / \mathrm{mL}$ TGF- $\beta 1$ (R\&D, MN) or $8 \mu \mathrm{M}$ BAPN (Sigma, MO) added for the 3 additional weeks.

\section{shRNA transduction}

pLKO.1-derived expression vectors for small interfering RNAs against $L O X, L O X L 1, L O X L 4$, and SMAD3, designed 
according to the RNAi consortium (TRC), were purchased from Sigma shRNA library. The sense strand of the short hairpin RNA (shRNA) are: LOX 5'-CCGG CTGCACAA TTTCACCGTATTACTCGAGTAATACGGTGAAATTG TGCAGTTTTTG-3' LOXL1 5'-CCGGACTATGTGCAAG CATCCACTTCTCGAGAAGTGGATGCTTGCACATAG TTTTTG-3' ' LOXL4 5'-CCGGAGAGTCAGATTTCTCC AACAACTCGAGTTGTTGGAGAAATCTGACTCTTTT TTG-3' SMAD3 5'-CCGGCCCAGCACATAATAACTTG GACTCGAGTCCAAGTTATTATGTGCTGGGTTTTT-

$3^{\prime}$. Plasmids encoding shRNA sequences $(1 \mu \mathrm{g})$ were cotransfected with $0.75 \mu \mathrm{g}$ psPAX2 and $0.25 \mu \mathrm{g}$ pMD2.G (Addgene, MA) into HEK293T cells. Supernatant of lentivirus was harvested and filtered through a $0.4-\mu \mathrm{m}$ filter (Millipore, MA) and then applied to HFF with $1 \mathrm{ng} / \mathrm{mL}$ polybrene (Sigma, MO) for $7 \mathrm{~h}$ followed by $2 \mu \mathrm{g} / \mathrm{mL}$ puromycin selection for 2 weeks.

\section{Atomic force microscopy (AFM)}

A 5- $\mu \mathrm{m}$ diameter borosilicate spherical tip (Novascan, IA) with a spring constant of $0.06 \mathrm{~N} / \mathrm{m}$ was used to determine elastic modulus on both the lower (stromal) and upper (epithelial) surfaces of hSEs and on the hDEs. Nine or more valid measurements were collected randomly across an area of 5-mm in diameter under force-contact mode on each side of these tissues. One tissue in each group from three independent experiments was used to measure the tissue stiffness by AFM.

\section{SHG/TPEF microscopy}

Images $(512 \times 512$ pixels; $600.0 \times 600.0 \mu \mathrm{m})$ were acquired at 3 - $\mu \mathrm{m}$ z-stacks (70 z-slices) by a Leica TCS SP2 confocal microscope equipped with a tunable titanium-sapphire laser (Spectra Physics, CA) and a water-immersion 25x objective. To collect SHG signal, an excitation wavelength of $800 \mathrm{~nm}$ and an emission range of $400 \pm 10 \mathrm{~nm}$ was used. TPEF signal was collected using a $525 \pm 25 \mathrm{~nm}$ emission filter at $755 \mathrm{~nm}$ excitation. Reflected signals were collected under epi-illumination mode for both TPEF and SHG imaging. The TPEF and SHG images were processed in MATLAB to obtain quantitative metrics of ECM microstructure. A weighted vector summation algorithm was used to determine the collagen fiber orientation by $2 \mathrm{D}$ variance [15]. The cumulative and mean TPEF signals were computed within the collagen-positive mask as measures of collagen cross-linking $[13,16]$.

\section{Hydroxyproline assay}

Hydroxyproline Assay kit (Cell Biolabs, CA) was used according to the manufacture's manual. Briefly, SASs were homogenized with PBS using a plastic pestle and hydrolyzed using $12 \mathrm{~N}$ hydrochloride acid at $120^{\circ} \mathrm{C}$ for $3 \mathrm{~h}$. They were then mixed with activated charcoal by vortexing and centrifuging at $10000 \mathrm{~g}$ for $5 \mathrm{~min}$. Supernatants were transferred into new tubes and incubated with chloramine $\mathrm{T}$ mixture for $30 \mathrm{~min}$ at room temperature, followed by an incubation with 4-(dimethylamino) benzaldehyde (DMAB) mixture for $90 \mathrm{~min}$ at $60^{\circ} \mathrm{C}$. The absorbance was read at $540-560 \mathrm{~nm}$ and hydroxyproline content was determined by comparison with a predetermined standard curve and normalized to the cell number indicated by DNA amount using Quant-iT PicoGreen dsDNA Assay kit (TheroFisher Scientific, IL).

\section{ELISA of cross-linked carboxy-terminal telopeptide of type I collagen (CTX-1)}

Human C Telopeptide of Type I Collagen (CTX-1) ELISA kit (MyBioSource, CA) was used according to the manufacture's manual. Briefly, SASs were homogenized with PBS using a plastic pestle on ice. Samples were centrifuged for $15 \mathrm{~min}$ at $1500 \mathrm{~g}$, and the supernatants were aliquoted and stored at $-80^{\circ} \mathrm{C}$ to avoid repeated freeze-thaw cycles. In total, $50 \mu \mathrm{L}$ of each sample in duplicates were incubated on a microtiter plate pre-coated with HPR conjugated CTX-1 antibody at $37^{\circ} \mathrm{C}$ for $1 \mathrm{~h}$. The enzyme-substrate reaction was terminated by adding $50 \mu \mathrm{L}$ of sulfuric acid solution, and the color change was measured spectrophotometrically at a wavelength of $450 \mathrm{~nm}$. The CTX-1 concentration in each sample was interpolated from the standard curve and normalized to total collagen content detected by hydroxyproline assay kit (Cell Biolabs, CA) using the same homogenates in PBS.

\section{Statistics}

Results were demonstrated as mean \pm SD of three replicates $(n=3)$ within the same group for all experiments in this study. Statistical analysis was performed using GraphPad Prism (GraphPad, SD). Nonparametric $t$ test (Mann-Whitney test) was used for comparison within two groups and one-way ANOVA with Tukey's posttest or twoway ANOVA with Bonferroni's posttest were used for multiple comparisons within more than two groups. $P<$ 0.05 was considered to be statistically significant.

\section{Results}

\section{hSEs mimic hallmarks of tissue fibrosis upon stimulation with TGF- $\beta 1$}

To mimic pro-fibrotic progression, hSEs were treated with TGF- $\beta 1$ at various doses $(1,2,5 \mathrm{ng} / \mathrm{mL})$ and compared with 
Fig. 1 TGF- $\beta 1$ induces profibrotic hallmarks including increased contractility, fibroblast activation and tissue stiffness in hSEs. a Schematic of hDEs and hSEs. b Representative images of hDEs and hSEs treated with 2 $\mathrm{ng} / \mathrm{mL}$ TGF- $\beta 1$. $\mathbf{c} \mathrm{qPCR}$ of COL1A2 and ACTA2 in HFFs isolated from $\mathrm{hDEs}$ and $\mathrm{hSEs}$ treated with $2 \mathrm{ng} / \mathrm{mL}$ TGF- $\beta 1$. d, e Immunohistochemistry staining of $\alpha$ SMA in hDEs (d) and hSEs (e) treated with TGF$\beta 1$ ( $2 \mathrm{ng} / \mathrm{mL})$. Myofibroblast percentage was calculated by counting $\alpha$ SMA-positive fibroblasts out of a total number of nuclei. Scale bar: $50 \mu \mathrm{m}$. $\mathbf{f}$ Expression of $K G F$ in HFF from hSEs and hDEs treated by $2 \mathrm{ng} /$ $\mathrm{mL}$ TGF- $\beta 1$. g AFM

measurements of hSEs and hDEs treated with TGF- $\beta 1$ (2 $\mathrm{ng} / \mathrm{mL}$ ) or vehicle control. Valid measurements $(>=9)$ were collected randomly under forcecontact mode. Error bars show mean $\pm \mathrm{SD}(n=3) .(* P<0.05$, $* * P<0.01, * * * P<0.001$, ns not significant)
A

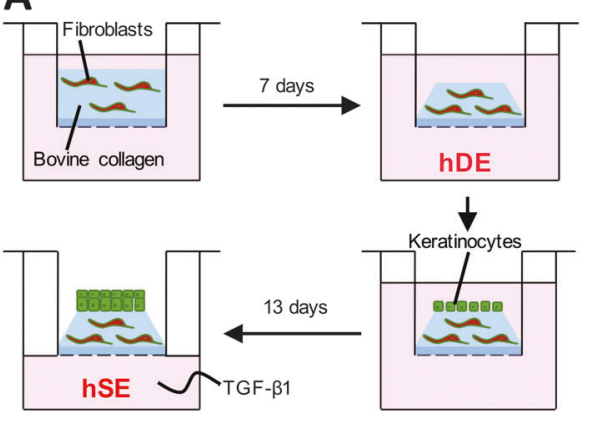

B

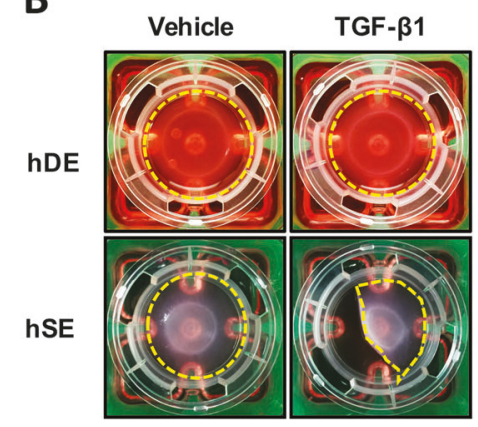

${ }_{A C T A Z}$

C

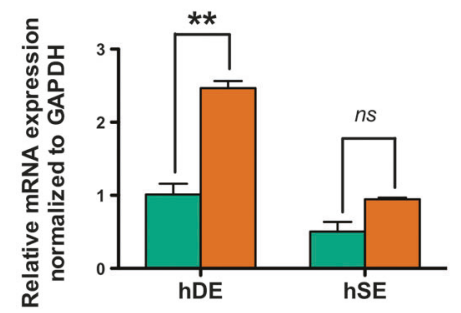

D
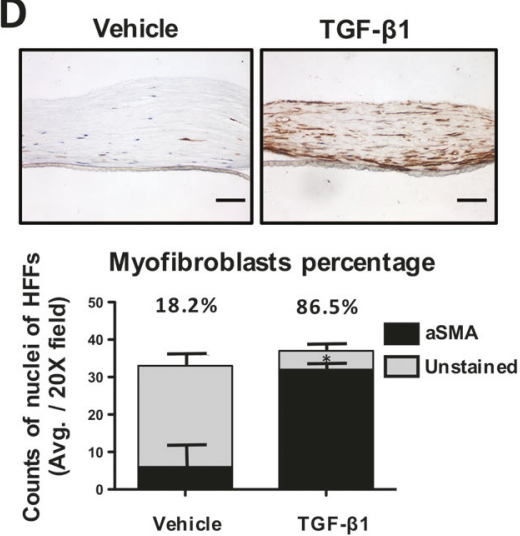

F

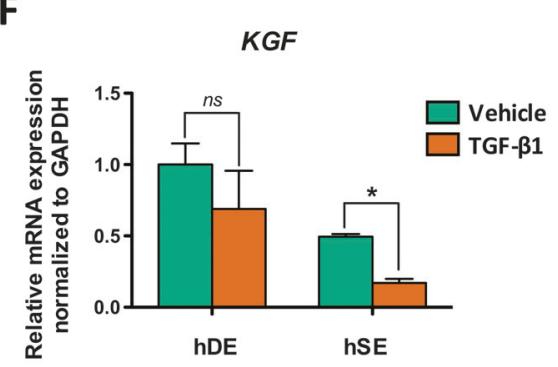

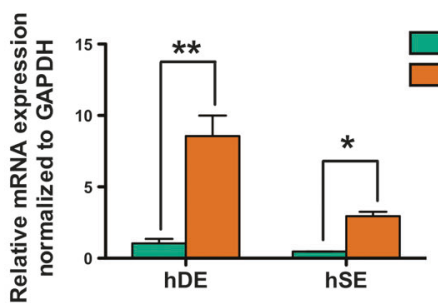

E
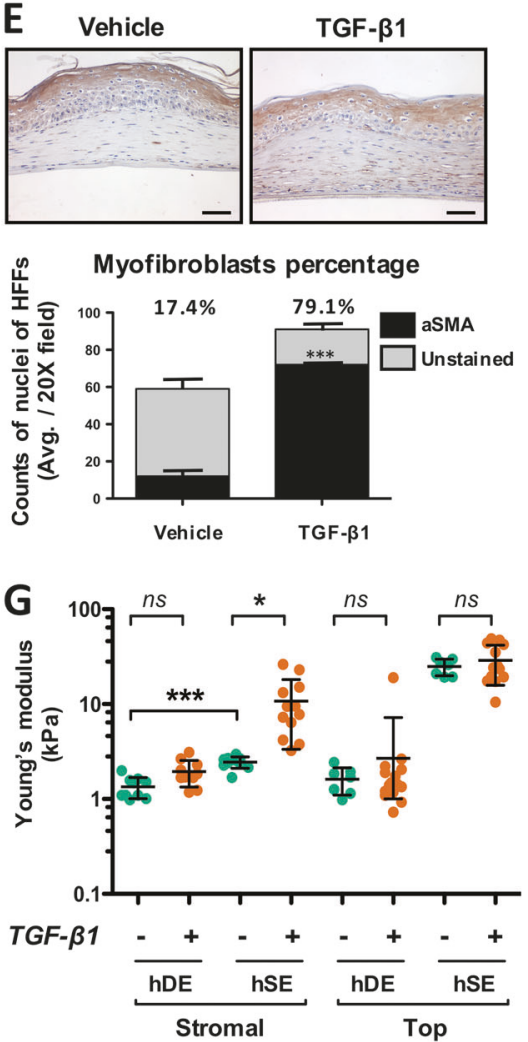

their responses in human dermal equivalents (hDEs), harboring human foreskin fibroblasts (HFFs), but grown without an overlying epithelium (Fig. 1a). Morphologic contraction was observed in hSEs after treatment with TGF$\beta 1$ in a dose-dependent manner, as seen by a continuous decrease in the relative tissue surface area with increasing dose of TGF- $\beta 1$ (S1A-B Figs and Fig. 1b). In contrast, no contraction of hDEs constructs was seen at any dose treatment of TGF- $\beta 1$ (S1A Fig and Fig. 1b). Additionally, a distinct gene profile of TGF- $\beta$-response was found in HFFs grown in hSEs compared with those cultured in $2 \mathrm{D}$ monolayer. Transcripts of TGF- $\beta 1$-targeted genes $(C T G F$, PAI-1, ACTA2) were consistently three to fourfold lower in hSEs than in 2D cultures at various dose treatment (S1C and 
D Figs). Notably, COL1A2 was increased significantly with $2 \mathrm{ng} / \mathrm{ml}$ TGF- $\beta 1$ in $2 \mathrm{D}$ but remained unchanged in hSEs (S1C-D Figs), as previously observed using in vivo skin fibrosis models [17, 18]. Thus, $2 \mathrm{ng} / \mathrm{ml}$ was selected for subsequent experiments to evaluate the TGF- $\beta$-response of stromal fibroblasts in hSEs compared with that in hDEs. Similar with findings in 2D cultured HFFs, both ACTA2 and COL1A2 were significantly increased by TGF- $\beta 1$ in hDEs (Fig. 1c). Myofibroblast activation was confirmed by immunohistochemical staining of $\alpha$ SMA in hSEs, showing an increase in fibroblasts positive for $\alpha$ SMA from $17.4 \%$ to $79.1 \%$, and was accompanied by an increased proliferation of HFFs (Fig. 1d). In hDEs, there was approximately half the number of HFFs ( $<40$ counted on cross-section), but a higher proportion of myofibroblasts than in hSEs (Fig. 1e). Furthermore, keratinocyte growth factor (KGF), a paracrine-acting, epithelial mitogen produced by stromal cells, was significantly decreased by TGF- $\beta 1$ only in hSEs but not in hDEs (Fig. 1f).

Skin stiffening is a hallmark of fibrotic progression. In light of this, the elastic modulus of both the top (epithelial) and bottom (stromal) surfaces of hSEs were measured by AFM to determine if changes in TGF- $\beta 1$-mediated gene expressions were associated with changes in tissue stiffness in hSEs. Measuring stiffness from the epithelial surface of hSEs did not yield differences between TGF- $\beta 1$ and vehicle treatment (Fig. 1g, Top). However, there was a fivefold increase in stiffness on the stromal surface of hSEs induced by TGF- $\beta 1$ (Fig. $1 \mathrm{~g}$, Stromal), which constitutes the region of skin where fibrosis occurs. Importantly, neither measurement from the top nor bottom surfaces of hDEs found an increased stiffness in response to TGF- $\beta 1$ (Fig. 1g), demonstrating that a well-developed epithelial layer was essential for TGF- $\beta 1$-mediated changes in stromal rigidity. Additionally, IL-13, known to be a pro-fibrotic cytokine that induces cutaneous fibrosis [19], modulated stromal tissues in a way similar to TGF- $\beta 1$, inducing tissue stiffness in the stroma of both $\mathrm{hDEs}$ and hSEs that was accompanied by an increase of myofibroblasts expressing $\alpha \mathrm{SMA}$ (S2 Fig).

Taken together, the epithelial-stromal interaction in the tissue microenvironment of hSEs provides a useful model for recapitulating hallmarks of skin fibrosis including tissue contraction, fibroblast activation, and tissue stiffness.

\section{Inhibition of LOXs modulates fibrotic phenotypes in hSEs}

It is known that fibrotic processes in the skin are characterized by excessive Type I collagen (COLI) deposition [20]. While TGF- $\beta 1$ did not increase the transcription of COL1A2 in hSEs (Fig. 1c), we hypothesized that collagen homeostasis was impaired by TGF- $\beta 1$ through the augmentation of posttranslational modification of COLI. To test this, we investigated the role of the LOXs in mediating collagen cross-linking by adding $\beta$-aminopropionitrile (BAPN), a small molecule inhibitor that is known to irreversibly block the activity of LOXs, either alone or in combination with TGF- $\beta 1$ to hSEs. Interestingly, the greatest degree of tissue contraction was found when BAPN and TGF- $\beta 1$ were added in combination compared with TGF- $\beta 1$ alone ( $25.6 \%$ vs. $60.3 \%$ of remaining tissue surface area) (Fig. 2a). This suggested that blocking collagen crosslinking resulted in a reduction of the resistance of hSEs to TGF- $\beta 1$-induced tissue contraction. Furthermore, BAPN led to a decreased stiffness in stromal tissues and this decrease was maintained even with the presence of TGF- $\beta 1$ (Fig. $2 b$ ), further indicating that collagen cross-linking is critical to maintain the mechanical properties of the stroma, corroborating the findings of previous studies [21, 22]. The unchanged COL1A2 showed that the mechanical property of hSEs was not associated with altered de novo synthesis of COLI by HFFs (Fig. 2c). However, the induction of ACTA2 seen in response to TGF- $\beta 1$ was greatly reduced by BAPN (Fig. 2c), indicating that the TGF- $\beta 1$-mediated contraction in hSEs is independent of the increased expression of ACTA2.

hSEs treated with TGF- $\beta 1$ in combination with BAPN demonstrated a loss of stromal tissue architecture characterized by the misalignment of HFFs being with collagen fibers in the stroma, in comparison with parallel oriented HFFs seen in other conditions (Fig. 2d, arrows). To further explore this, SHG/TPEF signals were collected to quantitatively assess modification of collagen microstructure. Collagen cross-linking was suppressed by BAPN in the presence of TGF- $\beta 1$ (Fig. 2e, TPEF and $2 \mathrm{f}$, red). In addition, the collagen fibers were less aligned in the presence of BAPN (Fig. 2d, e, variance), in line with previous findings that collagen fibers were less parallel in non-fibrotic skin [23]. The total amount of fibrillar collagen was significantly increased by TGF- $\beta 1$ but was attenuated by BAPN (Fig. 2e, SHG and $2 \mathrm{f}$, green and $2 \mathrm{~g}$ ), implying that blocking LOXsmediated cross-linking of collagen resulted in a decreased deposition of fibrillar collagen and further explained the dramatic tissue deformation induce by TGF- $\beta 1$ with the present of BAPN (Fig. 2a). These findings suggest that TGF- $\beta 1$-induced stiffness, contraction, and alterations in collagen organization via LOXs-mediated collagen crosslinking.

\section{LOXs activity is required for TGF- $\beta 1$-induced accumulation of de novo collagen}

The dermal tissue model of SASs, in which fibroblasts produce and assemble their endogenous ECM, was used to evaluate the de novo deposition of ECM by HFFs (Fig. 3a). 
A

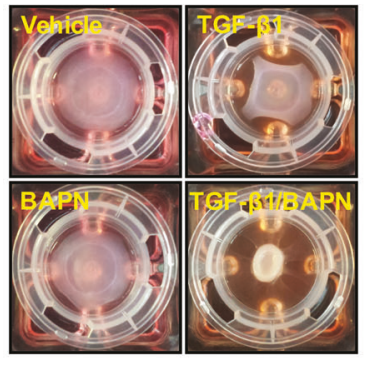

C

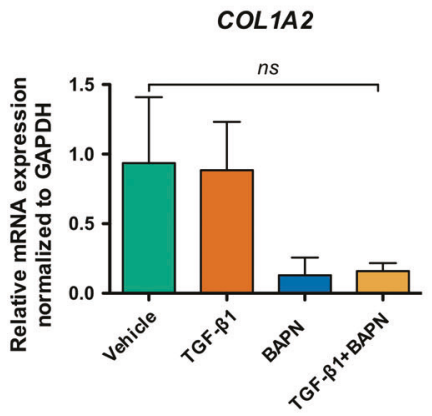

E

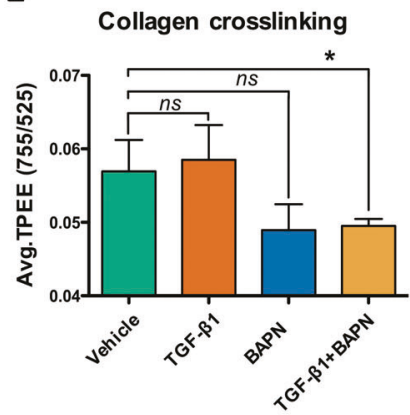

$\mathbf{F}$

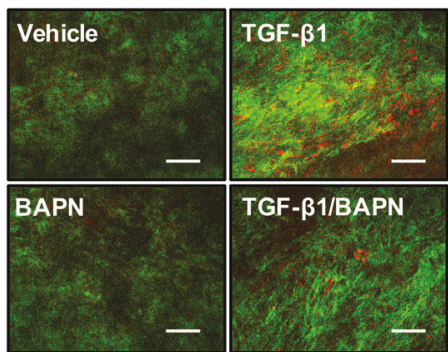

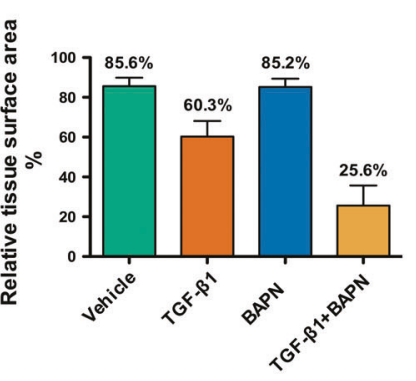
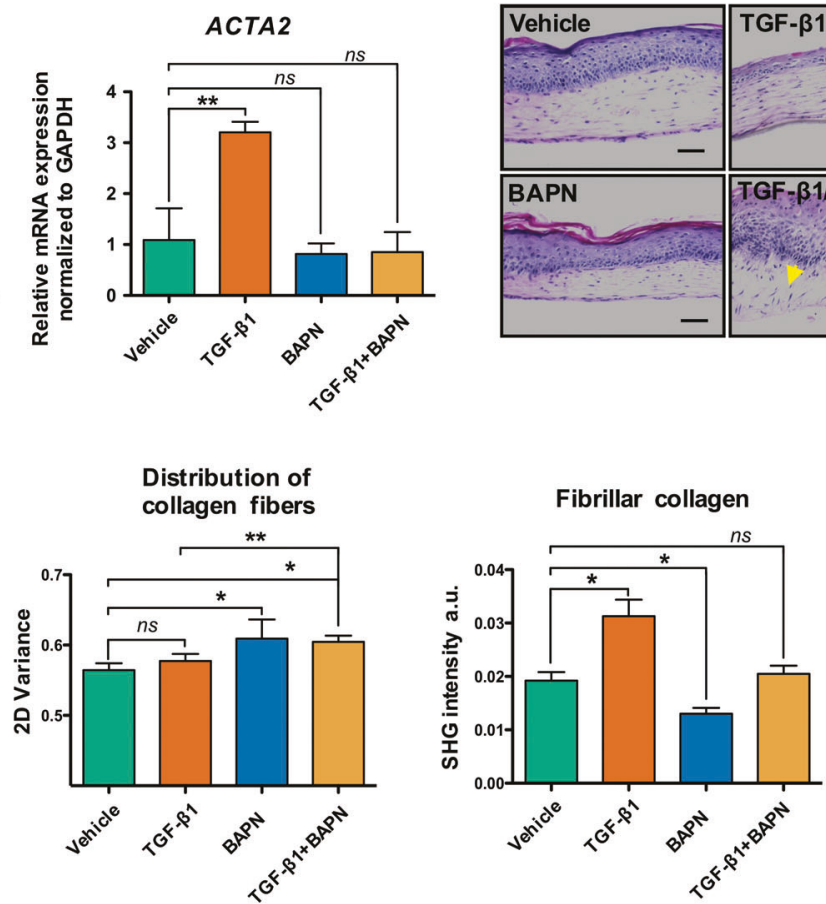

G

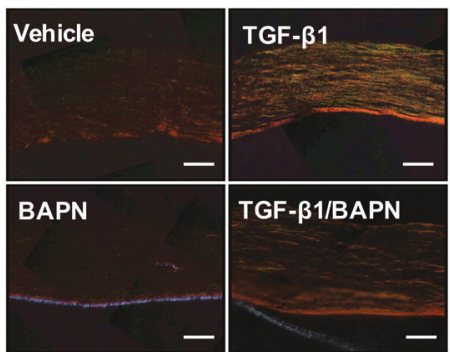

Fig. 2 Suppression of LOXs-mediated collagen cross-linking alters stromal stiffness and collagen organization in hSEs. hSEs were treated with $2 \mathrm{ng} / \mathrm{mL}$ TGF- $\beta 1$ or/and $8 \mu \mathrm{M}$ BAPN for 8 days. a Representative hSEs images and relative surface area that was calculated by comparing tissue surface to transwell membrane surface using Image J. b AFM measurement on the stromal surface of hSEs. Valid measurements $(>=9)$ were collected randomly under force-contact mode. c qPCR of COL1A2 and ACTA2 in fibroblasts. d H\&E staining. Arrows: fibroblasts alignment. Scale bar: $50 \mu \mathrm{m}$. e Collagen microstructures

SASs were treated with TGF- $\beta 1$ in the presence or absence of BAPN-formed nodules (Fig. 3b, arrow), which showed a

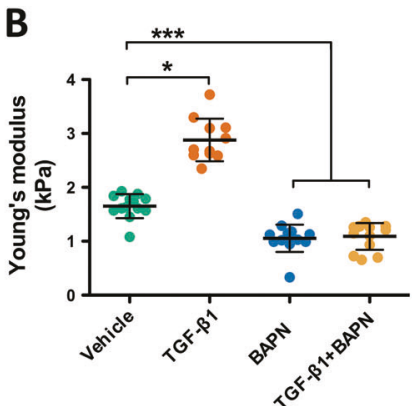

D

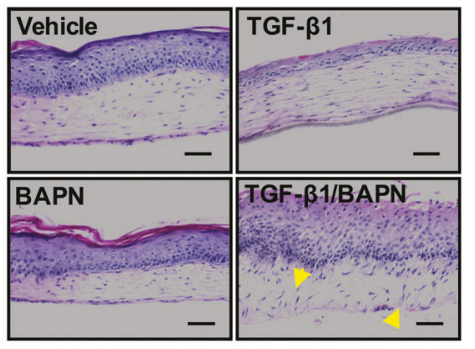

quantified by SHG/TPEF microscopy. f Representative images of SHG/TPEF microscopy. Green: SHG signals ( $800 \mathrm{~nm}$ excitation, 400 $\pm 10 \mathrm{~nm}$ emission); red: TPEF signals (755 nm excitation, $525 \pm 25 \mathrm{~nm}$ emission). Scale bar: $100 \mu \mathrm{m}$. g Representative images of Picrosirius red (PSR) staining. Yellow-orange birefringence: Type I collagen; blue: polycarbonate membrane. Scale bar: $50 \mu \mathrm{m}$. Error bars show mean $\pm \mathrm{SD}(n=3) . \quad(* P<0.05, * * P<0.01, \quad * * * P<0.001$, ns not significant)

homogeneous ECM with sparse HFFs that lacked the fibrillar structure seen in vehicle-treated and BAPN-treated 
A

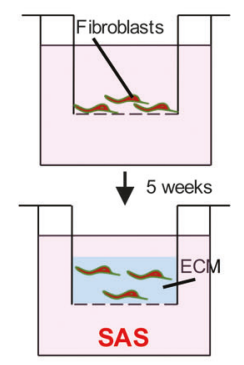

B

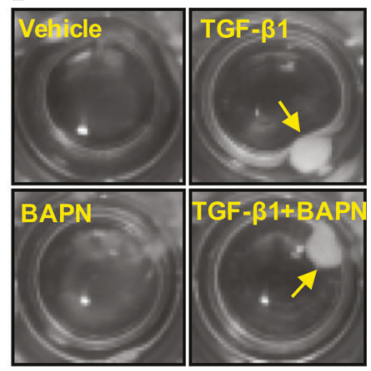

C

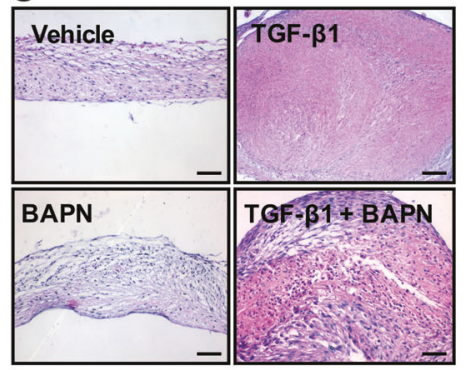

D

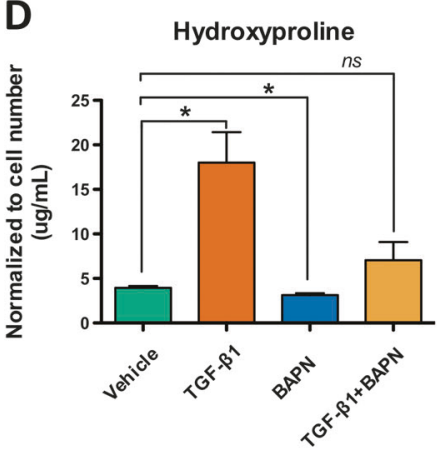

$\mathbf{F}$

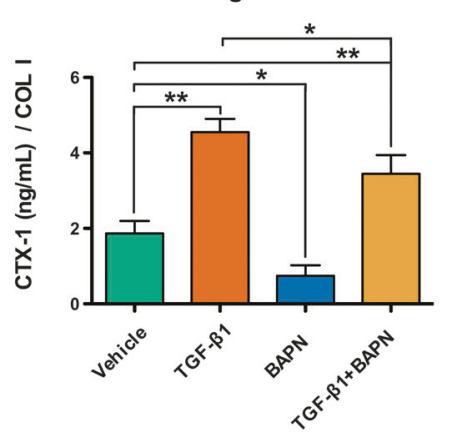

E

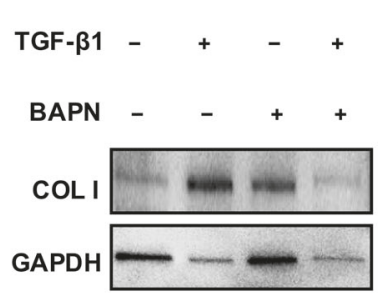

GAPDH $-\cdots$

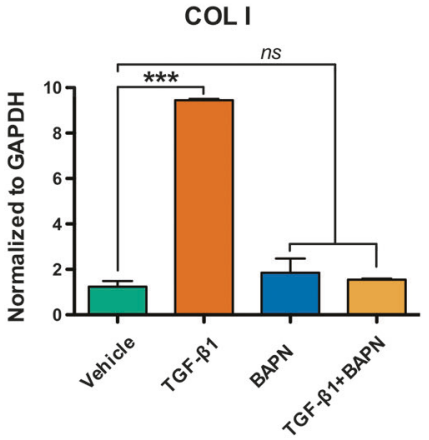

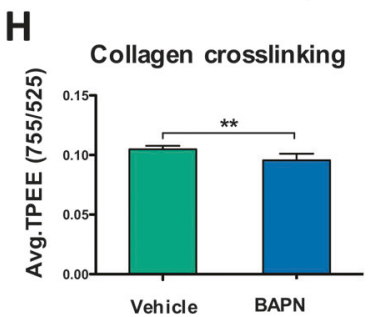

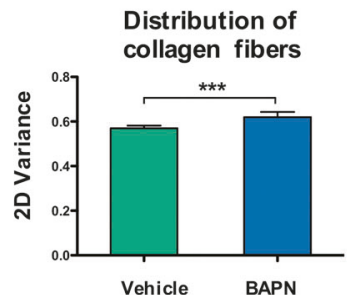

Vehicle BAPN Distribution of
collagen fibers

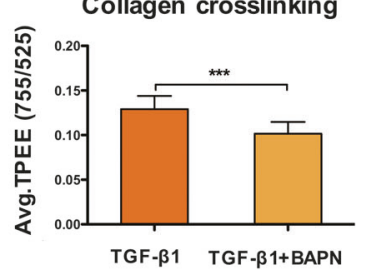

Collagen crosslinking
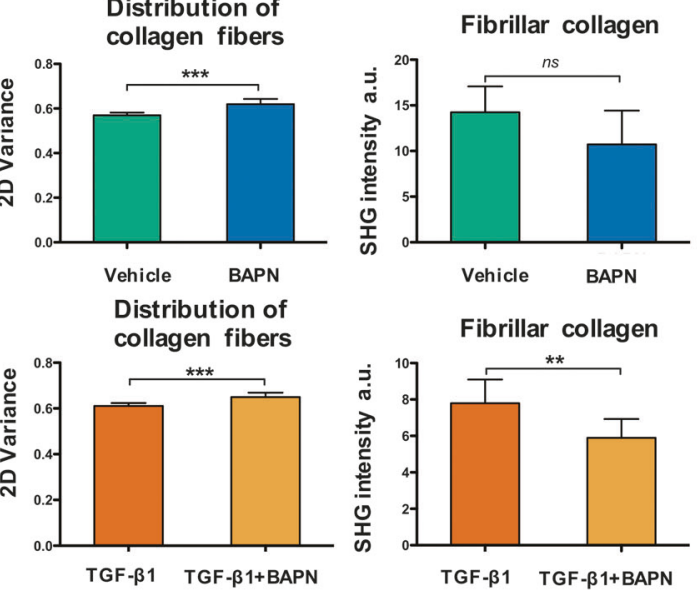

tissues (Fig. 3c). Total collagen content was significantly increased by TGF- $\beta 1$, but was inhibited with the addition of BAPN (Fig. 3d). Similarly, the elevated deposition and cross-linking of COLI were detected in the presence of
TGF- $\beta 1$, but tempered by exposure to BAPN (Fig. 3e, f). In line with these observations, the microstructural changes of collagen showed that BAPN decreased collagen crosslinking level (Fig. 3g, red and 3h, TPEF), alignment 
Fig. 3 Blocking LOXs-mediated cross-linking inhibits the de novo ECM accumulation in SASs. SASs were treated with TGF- $\beta 1(2 \mathrm{ng} /$ $\mathrm{mL}$ ) or/and $8 \mathrm{uM}$ BAPN for 3 weeks. a Schematic of SASs. b Representative SASs images. Arrows: nodule formation. c H\&E staining. Scale Bar: $50 \mu \mathrm{m}$. d Hydroxyproline assay of whole tissue lysate, normalized to total cell number. e Protein levels of COLI by western blot ( $200 \mathrm{kDa})$ and normalized to GAPDH ( $37 \mathrm{kDa})$. f COLI cross-linking level measured by CTX-I ELISA kit and normalized to total protein amount detected in (d). g Representative images of SHG/ TPEF microscopy. Green: SHG signals ( $800 \mathrm{~nm}$ excitation, $400 \pm 10$ $\mathrm{nm}$ emission); red: TPEF signals (755 nm excitation, $525 \pm 25 \mathrm{~nm}$ emission). Scale bar: $100 \mu \mathrm{m}$. h Levels of collagen cross-linking (TPEF), alignment (2D variance) and the total amount collagen (SHG) measured multiphoton (SHG/TPEF) microscopy. Error bars show mean $\pm \mathrm{SD}(\mathrm{n}=3) . \quad(* P<0.05, * * P<0.01, * * * P<0.001$, $\mathrm{ns}$ not significant)

(Fig. 3h, 2d, variance), and total collagen amount (Fig. 3g, green and $3 \mathrm{~h}, \mathrm{SHG})$. Thus, blocking LOXs by BAPN interferes with the structural organization of collagen, and limits the TGF- $\beta$-induced collagen deposition in a stromalike tissue.

In addition to collagen, other ECM components were also found to be upregulated by TGF- $\beta 1$. For example, both transcriptional and protein levels of EDA-fibronectin (EDAFn) splice isoform were strongly increased with TGF- $\beta 1$ stimulation (S3 Fig), suggesting that EDA-Fn may play an important role in de novo ECM deposition and organization.

\section{TGF- $\beta 1$-induced collagen remodeling mediated by LOXL4 in both hSEs and SASs}

To further explore mechanisms of LOXs-mediated collagen cross-linking, expressions of specific isoforms were analyzed in HFFs harbored by hSEs. LOX, LOXL1, and LOXLA were increased by 3.1-, 1.7-, and 2.3-fold, respectively, in response to TGF- $\beta 1$, whereas LOXL2 and LOXL3 were unchanged (Fig. 4a). In light of this, HFFs were transduced by lentivirus encoding shRNA to knockdown $L O X, L O X L 1$, and LOXL4 (Fig. 4b). We found that stromal stiffness was significantly decreased in hSEs harboring HFFs with the suppression of LOX or LOXL4 ((Fig. 4c). These findings indicate that $L O X$ - and $L O X L 4$-mediated cross-linking is important in maintaining stromal stiffness in tissues that mimic human skin.

Next, the roles of $L O X, L O X L 1$, and $L O X L 4$ were studied in the de novo synthesized ECM by SASs. The inhibition of LOXL4 completely blocked the TGF- $\beta 1$-induced formation of nodules, whereas it was persistently seen in tissues containing HFFs upon knockdown LOX or LOXL1 (Fig. 4d, arrows). Importantly, TGF- $\beta 1$-induced collagen was significantly suppressed by knocking down $L O X, L O X L 1$, or LOXL4 (Fig. 4e, f). However, only blocking LOXL4 was sufficient to inhibit TGF- $\beta 1$-induced COLI cross-linking
(Fig. 4g). Such similar inhibitory effects were seen in SASs harboring HFFs upon knockdown of SMAD3, a known mediator of TGF- $\beta$-induced fibrosis, where TGF- $\beta 1$ induced production of collagen was completely suppressed (S4B-D Figs). However, knockdown of SMAD3 failed to block nodule formation (S4A Fig), implying that ECM components other than collagen might be regulated by TGF- $\beta 1$ through non-Smad3 pathways. For instance, EDAFn was continuously increased by TGF- $\beta 1$ in SASs that harbored HFFs with knockdown of SMAD3 or any LOXs isoforms (S5 Fig), suggesting that LOXs-mediated ECM modification was more restricted to collagen and independent from SMAD3 regulated by TGF- $\beta 1$. Taken together, these suggest LOXL4 might be a potential target to inhibit TGF- $\beta 1$-induced stromal stiffness and collagen accumulation, both of which appear to be associated with the collagen cross-linking level.

\section{Modification of fibrotic phenotypes by targeting LOXL4 in SSCDFs}

To further establish the clinical relevance of our skin-like tissue models, we tested the role of LOXL4 in a connective tissue disease characterized by tissue fibrosis. We incorporated SSc patient-derived fibroblasts (SScDFs) in hSEs and found elevated transcripts of LOXL4 in comparison with normal dermal fibroblasts (NDFs) (Fig. 5a). The upregulation of LOXL4 mRNA was also observed in our previous finding [24] based on DNA microarray analyses of skin biopsies from 24 SSc patients and six healthy controls (S6 Fig). By performing knockdown of LOXL4 in SScDFs, we found decreased matrix stiffness in hSEs as determined by AFM (Fig. 5b). In addition, there was less matrix contraction with suppression of LOXL4 in SScDFs as seen by the enlarged plateau measured on the epithelial surface of hSEs (Fig. 5c). Interestingly, a significant increase of ACTA2 induced by TGF- $\beta 1$ was found in SScDFs while COL1A2 stayed almost unchanged (Fig. 5d, e), as was seen in previous findings in HFFs (Figs $1 \mathrm{c}$ and 2c). Similar to HFFs treated with BAPN in the presence of TGF- $\beta 1$, we found a significant reduction of both ACTA2 and COL1A2 induced by TGF- $\beta 1$ in SScDFs with LOXL4-KD (Figs 5d, e, and 2c), suggesting that TGF- $\beta 1$-mediated fibroblasts activation could be suppressed by targeting LOXL4 in SScDFs. Furthermore, de novo ECM of SScDFs was assessed using SASs. TGF$\beta 1$-induced collagen aggregation and accumulation was demonstrated by the nodule formation, which was blocked by inhibition of LOXL4-mediated collagen cross-linking (Fig. 5f, g). Taken together, these results demonstrated that TGF- $\beta$ response modulated by LOXLA could be studied in our tissue models harboring primary cells from SSc patients. This suggests the future translational use of 
A

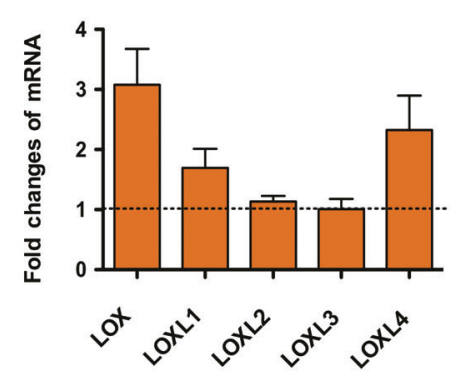

D

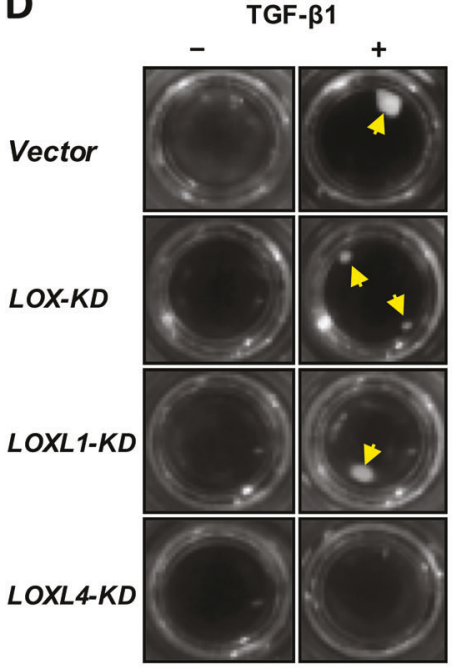

$\mathbf{F}$
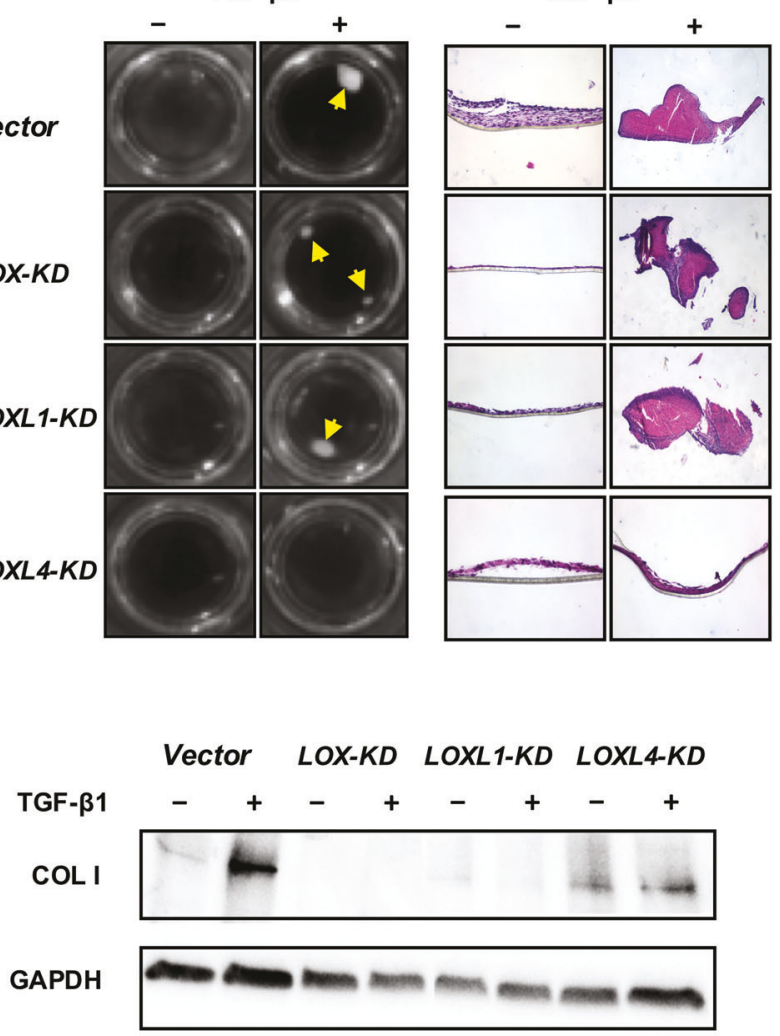

B

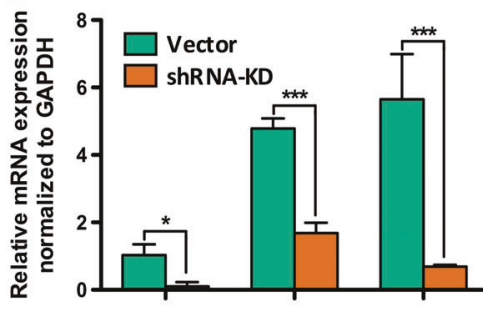

$\mathrm{s}^{+}$

$v^{+x^{2}}$

$0^{+x^{x}}$
C

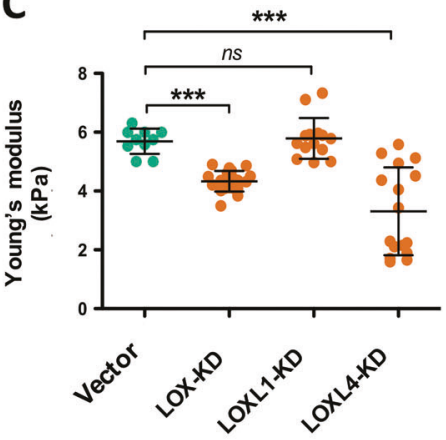

Hydroxyproline

E

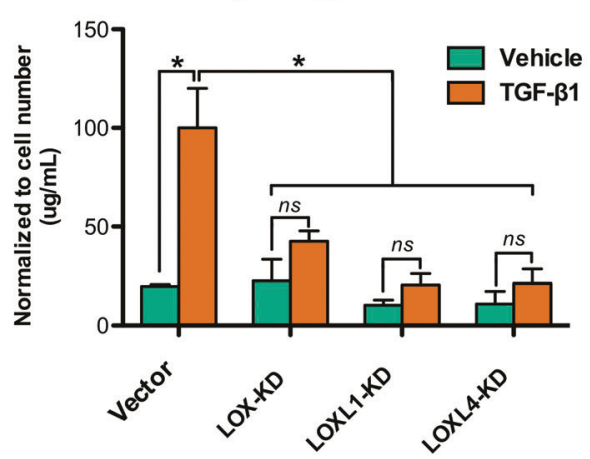

G Crosslinking of COL I

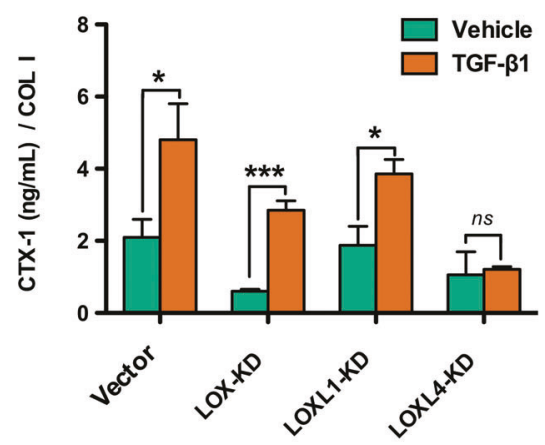

Fig. 4 Knockdown of specific LOXs suppresses TGF- $\beta 1$-mediated induction of stromal stiffness in hSEs and de novo COLI in SASs. a qPCR of $L O X$ and LOXL1-4 in HFFs isolated from hSEs treated with $2 \mathrm{ng} / \mathrm{mL}$ TGF- $\beta 1$. b qPCR of LOX, LOXL1 and LOXL4 in HFFs transduced with $L O X$-shRNA, LOXL1-shRNA and LOXL4-shRNA respectively compared to vector control. c AFM measurements of Young's modulus on the stromal surface of hSEs. Valid measurements $(>=9)$ were collected randomly under force-contact mode. d

hSEs and SASs to investigate pathways and mechanisms of fibrosis.

\section{Discussion}

There is a compelling need to understand the underlying mechanisms linked to fibrogenic phenotypes in human
Representative images and H\&E staining of SASs treated with $2 \mathrm{ng} /$ $\mathrm{mL}$ TGF- $\beta 1$. Arrows: nodule formation. e Collagen content measured by hydroxyproline assay of SASs lysate in PBS. Signal was normalized to total cell number. $\mathbf{f}$ Western blot bands showed the protein levels of COLI $(200 \mathrm{kDa})$ and GAPDH $(37 \mathrm{kDa})$. g COLI crosslinking level of SASs lysate in PBS using CTX-I ELISA kit and normalized to total collagen amount detected in (e). Error bars show mean $\pm \mathrm{SD}(n=3) .(* P<0.05, * * * P<0.001, \mathrm{~ns}$ not significant $)$

tissues. However, the shortage of accurate animal models that mimic fibrogenic processes have been a rate-limiting factor for basic science and preclinical studies [25]. In recent years, novel tissue engineering approaches have resulted in human, 3D tissue-based, in vitro tissue models with high fidelity to human disease, that can shorten preclinical drug development timelines, reduce testing in animals and limit drug failure rates that can improve the 

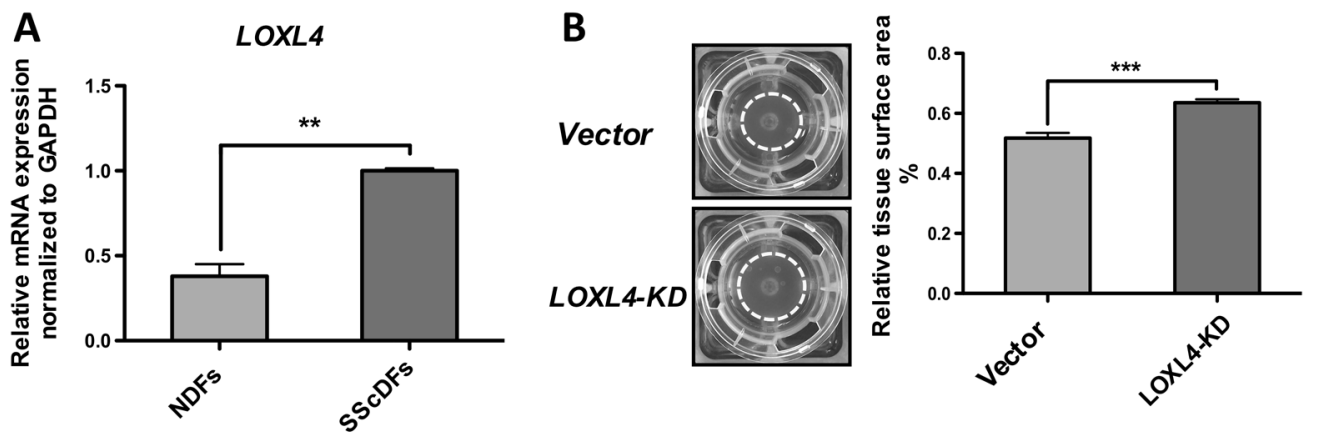

C

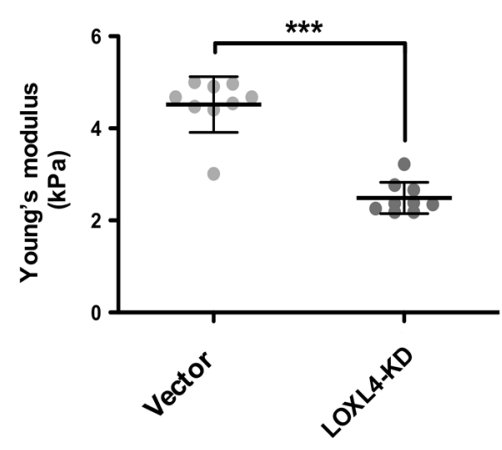

E
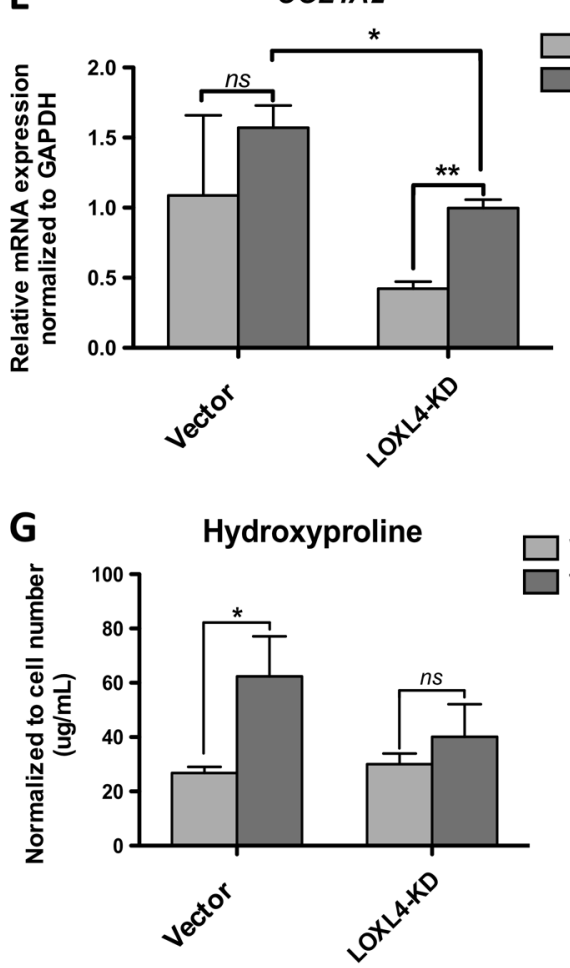

Fig. 5 Modulating fibrotic phenotypes in hSEs and SASs incorporated with SScDFs by targeting LOXL4. SScDFs transduced with lentivirus encoding the shRNA targeting LOXL4 were treated with $2 \mathrm{ng} / \mathrm{mL}$ TGF- $\beta 1$ in hSEs and SASs. a qPCR of LOXLA in NDFs and SScDFs. b AFM measurement on the stromal surface of hSEs. Valid measurements $(>=9)$ were collected randomly under force-contact mode. c Representative hSEs images and relative surface area that was
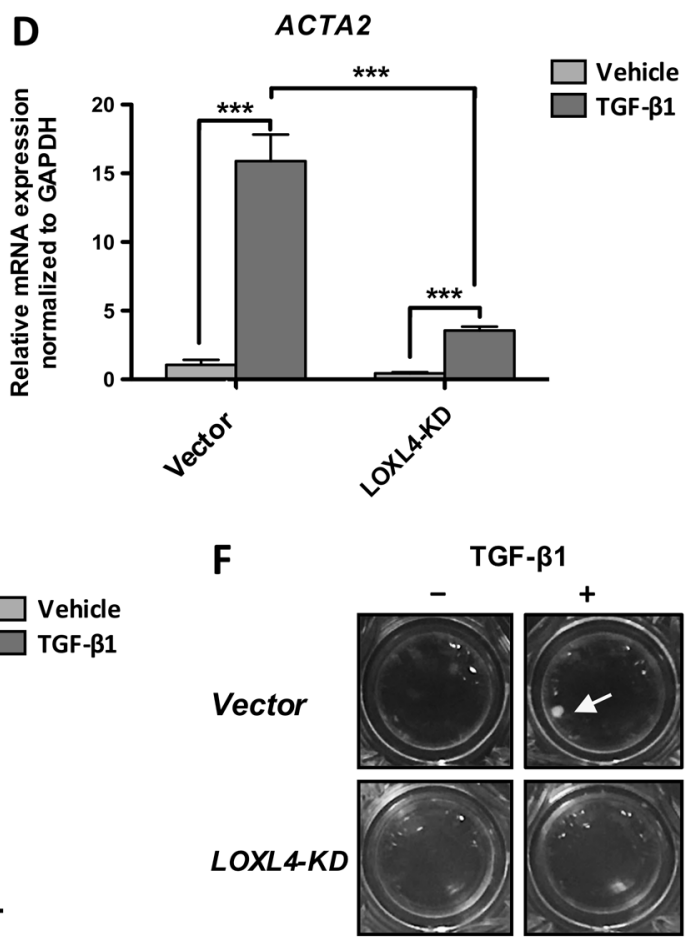

calculated by comparing surface of tissue plateau to transwell membrane surface using Image J. d, e qPCR of ACTA2 and COL1A2 in SScDFs dissociated from hSEs. f Representative images of SASs. Arrows: nodule formation. g Hydroxyproline assay of SASs lysate. Signal was normalized to total cell number. Error bars show mean \pm $\mathrm{SD}(n=3) .(* P<0.05, * * P<0.01, * * * P<0.001$, ns not significant $)$ 
Table 1 Prominent features of animal models and in vitro 3D models of fibrosis

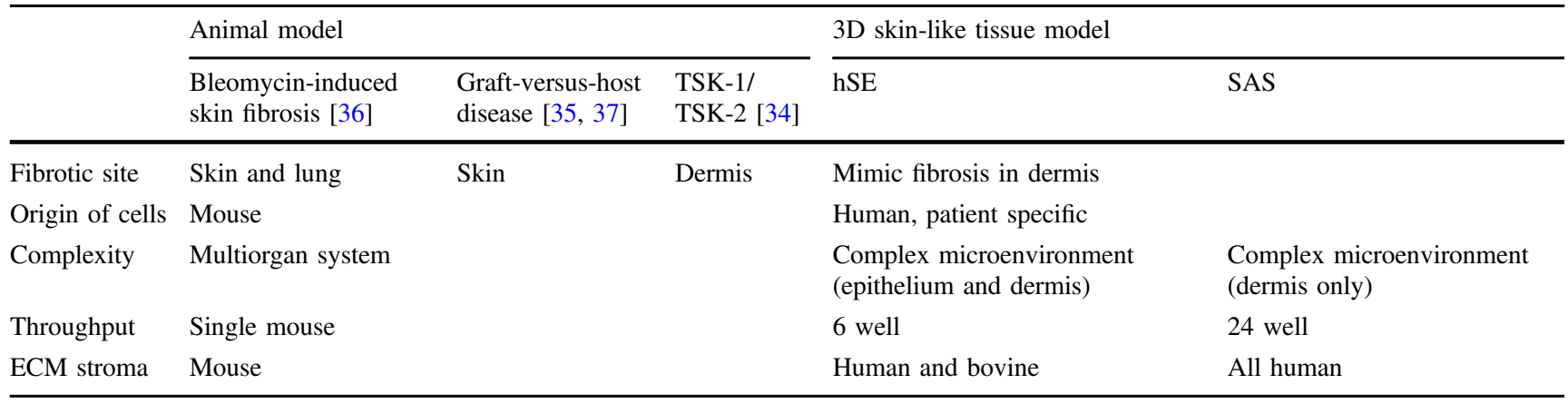

Lysyl oxidase enzymes mediate TGF- $\beta 1$-induced fibrotic phenotypes in human skin-like tissues

development process for new and effective disease therapeutics [26, 27]. To fill gaps between these existing models needed to study fibrosis, we have developed 3D tissue models of human skin that can mimic the complex cell interactions that more closely simulate fibrogenic processes and phenotypes as they occur in humans [28-33]. Table 1 lists the most commonly used mouse models [34-37] to study fibrosis and compares their prominent features to human, 3D tissue-based, in vitro models (hSE and SAS).

The data in this study indicate that hSEs and SASs provide consistent and reproducible findings upon TGF- $\beta 1$ stimulation, including increased tissue contractility and stiffness, fibroblast activation as determined by $\alpha \mathrm{SMA}$ staining, as well as altered ECM deposition and structure. Mediators of fibrosis (TGF- $\beta 1$ and IL-13) result in alteration of collagen cross-linking mediated by LOXs, modifying the mechanical properties of the ECM. Whereas the opposite effects were achieved in 3D tissues by inhibiting LOXmediated collagen cross-linking via BAPN. Furthermore, analyses of the LOXs family members using shRNA knockdown identify LOXL4 as the key enzyme mediating these fibrotic phenotypes. Therefore, targeting of LOXL4 therapeutically may reduce fibrosis in multiple diseases. By fabricating bioengineered tissues that recapitulate fibrotic phenotypes in a biologically relevant system, we provide physiologically relevant tissue models in which to identify pathways driving pathogenesis (e.g., skin stiffness and thickening), which can be used to test the efficacy of drugs that target fibrotic pathways.

In light of our findings, we have further established the utility of 3D human skin-like tissues as a paradigm for studying rare fibrotic skin diseases, such as that found in the systemic autoimmune disease, SSc. Currently, there are no FDA-approved therapies for SSc. Skin fibrosis is a hallmark of SSc, and prior studies have indicated that SSc dermal fibroblasts $(\mathrm{SScDFs})$ grown in $2 \mathrm{D}$ culture poorly recapitulate the phenotype found in SSc skin biopsies [38, 39]. We have also identified this difference in our tissue system as seen by elevated COL1A2 expression in $2 \mathrm{D}$ monolayer compared with unchanged levels in 3D tissues such as hSEs (S1C and D Figs). Thus, there is a desperate need for tissuebased, in vitro model systems that accurately recapitulate the SSc phenotypes observed in patients. Here, we extend our observations on LOXL4 in 3D tissues made from HFFs to tissues that incorporate SScDFs. hSEs made from SScDFs have a approximatelythreefold increase in stromal stiffness (Young's modulus of $\sim 5 \mathrm{kPa}$, Fig. $5 \mathrm{c}$ ) compared with hSEs made from HFFs (Young's modulus of $\sim 2 \mathrm{kPa}$, Fig. 2b), consistent with the increased level of dermal stiffness observed both in bleomycin-treated mice model and SSc patients [40, 41]. Knockdown of LOXL4 normalizes the stiffness of SScDFs in hSEs to levels similar to that found in tissues incorporating HFFs (Figs $2 b$ and $5 c$ ), while reducing expression of ACTA2 and COL1A2 (Figs 5d, e). This supports previous observations that regulation of ECM gene expression depends on the tissue microenvironment $[17,18]$, suggesting that mechanistic studies of cutaneous fibrosis also need to be investigated in tissues that more closely mimic in vivo physiologic conditions. While more work is needed to determine the utility of hSEs and SASs for studying SSc, this study provides confidence that such a system will recapitulate the molecular processes observed in SSc skin biopsies.

The ECM composition of fibrotic skin and its corresponding mechanical properties are dynamically changing as a result of ECM remodeling. We have previously demonstrated the value of SHG/TPEF microscopy as a nondestructive method for real-time monitoring of ECM structure, including collagen fiber organization, content, and cross-linking by comparing these optical biomarkers with conventional analysis of collagen composition and crosslinking $[13,15,16]$. Quantitative comparisons of SHG and TPEF signals between different samples require the samples to have similar optical properties including scattering and absorption [42, 43]. However, TGF- $\beta 1$ induces SASs to form highly scattering nodules, changing significantly the excitation and collection light paths and resulted in different baselines for both SHG and TPEF signals between flat and 
contracted tissues. Therefore, we compared groups of SASs with similar optical properties: vehicle vs. BAPN and TGF$\beta 1$ vs. TGF- $\beta 1+$ BAPN. When such differences are appropriately considered, the integration of SHG/TPEF microscopy with gene expression and mechanical properties of 3D skin models leads to a powerful platform to monitor fibrotic changes in human skin.

TGF- $\beta$ is a critical mediator of fibrotic responses, known to induce fibroblast synthesis, assembly, and contraction of ECM. Thus, a better understanding of how the TGF- $\beta$ signaling pathway regulates downstream targets linked to skin fibrosis, such as the activity of LOXs during skin fibrosis, can fill a gap in our understanding of TGF- $\beta$ associated, pro-fibrotic progression. It has been reported that the physical properties of the collagenous ECM, rather than the amount of collagenous structures within a tissue, are linked to loss of tissue function in tissues or organs affected by fibrosis [44]. Therefore, we studied how covalent cross-linking of COLI, mediated by LOXs, can modify the properties of 3D tissues in response to fibrotic stimuli. We found that BAPN, an inhibitor of all LOXs [45], blocked the TGF- $\beta 1$-mediated increase in stromal stiffness and was linked to suppression of $\alpha \mathrm{SMA}$, which in turn induced a dramatic alteration in the stromal morphology of hSEs. Previously, several knockout mouse models revealed that LOX and LOXL1-3 played important roles during embryonic development and their absence generated abnormal phenotypes due to deficiencies in ECM [46-49]. Additionally, these studies indicated that circulating level of LOX is an important biomarker for fibrosis [50]. By further dissecting the role of specific LOXs isoforms ( $L O X$, $L O X L 1$, and LOXL4) regulated by TGF- $\beta 1$, we have found that suppression of LOXL4 in HFFs and SScDFs generated a lower stiffness in hSEs and blocked TGF- $\beta 1$-induced ECM aggregation in SASs. This indicates that LOXL4 may play a pivotal role in the regulation of ECM homeostasis and could serve as an important mediator of cutaneous fibrosis.

Further understanding of ECM components that are altered in tissue fibrosis, such as COLI and EDA-Fn, will be critical to discovering pathways to treat this condition. For example, COLI is regulated by TGF- $\beta$ in a Smad-dependent manner in human skin fibroblasts [51, 52]. In contrast, Smad-independent pathways regulate EDA-Fn-associated fibroblast activation through integrin binding in cutaneous fibrosis [53, 54]. Consistent with these previous findings, we observed that the suppression of SMAD3 led to a reduction of TGF- $\beta 1$-induced COLI while deposition of EDA-Fn was sustained at a high level in response to TGF$\beta 1$. In addition, the knockdown of $L O X, L O X L 1$, and LOXL4 led to the suppression of TGF- $\beta 1$-induced COLI deposition, suggesting specific LOXs isoforms may be responsible for Smad3-mediated accumulation of COLI. As it was recently shown that $L O X$ expression can be regulated by $\alpha 1 \beta 2$ integrin binding to COLI, there seems to be an intriguing feedback loop between LOXs and COLI that warrants further study in the context of fibrosis [55].

In summary, our findings demonstrate that two distinct yet complementary 3D skin models (hSEs and SASs) provide biologically relevant insights into the role of TGF- $\beta 1$ induced and LOXs-mediated processes that are central to dermal fibrosis in human diseases. Future studies can exploit the utility of these tissues by increasing the tissue complexity by incorporating endothelial and immune cells or transplanting these tissues onto immune-deficient mice, as we have previously shown to create humanized-animal models of tissue fibrosis that can provide insights into skin fibrosis $[10,30,56]$.

Acknowledgements This research was funded by a grant from Biogen. In addition, a seed grant from Tufts Collaborates funded part of this research. Mengqi Huang received support from the China Scholarship Council to perform this work. We would like to thank the CMDB program, Sackler School of Graduate Biomedical Sciences at Tufts University, and Scleroderma foundation for their support. We thank Ryan Imbriaco for his technical assistance in the preparation of this manuscript and Gilad Cohen, Lev Brown, and Kamar Reda for their help with tissue imaging.

\section{Compliance with ethical standards}

Conflict of interest The authors declare that they have no conflict of interest.

\section{References}

1. Clements PJ, Hurwitz EL, Wong WK, et al. Skin thickness score as a predictor and correlate of outcome in systemic sclerosis: highdose versus low-dose penicillamine trial. Arthritis Rheum. 2000;43:2445-54.

2. Steen VD, Medsger TA Jr. Improvement in skin thickening in systemic sclerosis associated with improved survival. Arthritis Rheum. 2001;44:2828-35.

3. Ehrlich HP, Allison GM, Leggett M. The myofibroblast, cadherin, alpha smooth muscle actin and the collagen effect. Cell Biochem Funct. 2006;24:63-70.

4. Rosin NL, Agabalyan N, Olsen K, et al. Collagen structural alterations contribute to stiffening of tissue after split-thickness skin grafting. Wound Repair Regen. 2016;24:263-74.

5. Kendall RT, Feghali-Bostwick CA. Fibroblasts in fibrosis: novel roles and mediators. Front Pharmacol. 2014;5:123.

6. Hinz B. Tissue stiffness, latent TGF-betal activation, and mechanical signal transduction: implications for the pathogenesis and treatment of fibrosis. Curr Rheumatol Rep. 2009;11: 120-6.

7. Philip T, Debashree S, Manish B. Two functions of lysyl oxidases: extracellular matrix maturation and cell proliferation. FASEB J. 2015;29(1_supplement):570.518

8. Clarke DL, Carruthers AM, Mustelin T, et al. Matrix regulation of idiopathic pulmonary fibrosis: the role of enzymes. Fibrogenes Tissue Repair. 2013;6:20.

9. Sethi A, Wordinger RJ, Clark AF. Gremlin utilizes canonical and non-canonical TGFbeta signaling to induce lysyl oxidase (LOX) 
genes in human trabecular meshwork cells. Exp Eye Res. 2013;113:117-27.

10. Carlson MW, Alt-Holland A, Egles C, et al. Three-dimensional tissue models of normal and diseased skin. Curr Protoc Cell Biol. 2008; Chapter 19:Unit19. 19

11. Matsusaki M, Fujimoto K, Shirakata Y, et al. Development of full-thickness human skin equivalents with blood and lymph-like capillary networks by cell coating technology. J Biomed Mater Res A. 2015;103:3386-96.

12. Maione AG, Smith A, Kashpur O, et al. Altered ECM deposition by diabetic foot ulcer-derived fibroblasts implicates fibronectin in chronic wound repair. Wound Repair Regen. 2016;24:630-43.

13. Marturano JE, Xylas JF, Sridharan GV, et al. Lysyl oxidasemediated collagen crosslinks may be assessed as markers of functional properties of tendon tissue formation. Acta Biomater. 2014;10:1370-9.

14. Proia DA, Kuperwasser C. Reconstruction of human mammary tissues in a mouse model. Nat Protoc. 2006;1:206-14.

15. Quinn KP, Georgakoudi I. Rapid quantification of pixel-wise fiber orientation data in micrographs. J Biomed Opt. 2013;18: 046003.

16. Quinn KP, Sullivan KE, Liu Z, et al. Optical metrics of the extracellular matrix predict compositional and mechanical changes after myocardial infarction. Sci Rep. 2016;6:35823.

17. Nakasaki M, Hwang Y, Xie Y, et al. The matrix protein Fibulin-5 is at the interface of tissue stiffness and inflammation in fibrosis. Nat Commun. 2015;6:8574.

18. Zigrino P, Brinckmann J, Niehoff A, et al. Fibroblast-derived MMP-14 regulates collagen homeostasis in adult skin. J Invest Dermatol. 2016;136:1575-83.

19. Oh MH, Oh SY, Yu J, et al. IL-13 induces skin fibrosis in atopic dermatitis by thymic stromal lymphopoietin. J Immunol. 2011;186:7232-42.

20. van der Slot-Verhoeven AJ, van Dura EA, Attema J, et al. The type of collagen cross-link determines the reversibility of experimental skin fibrosis. Biochim Biophys Acta. 2005;1740:60-67.

21. Grover CN, Gwynne JH, Pugh N, et al. Crosslinking and composition influence the surface properties, mechanical stiffness and cell reactivity of collagen-based films. Acta Biomater. 2012;8:3080-90.

22. Lin S, Gu L. Influence of crosslink density and stiffness on mechanical properties of type I collagen gel. Mater (Basel). 2015;8:551-60.

23. Lee R, Perry B, Heywood J, et al. Caveolin-1 regulates chemokine receptor 5-mediated contribution of bone marrow-derived cells to dermal fibrosis. Front Pharmacol. 2014;5:140.

24. Milano A, Pendergrass SA, Sargent JL, et al. Molecular subsets in the gene expression signatures of scleroderma skin. PLoS One. 2008;3:e2696.

25. Marangoni RG, Varga J, Tourtellotte WG. Animal models of scleroderma: recent progress. Curr Opin Rheumatol. 2016;28: 561-70.

26. Esch EW, Bahinski A, Huh D. Organs-on-chips at the frontiers of drug discovery. Nat Rev Drug Discov. 2015;14:248-60.

27. Fabre KM, Livingston C, Tagle DA. Organs-on-chips (microphysiological systems): tools to expedite efficacy and toxicity testing in human tissue. Exp Biol Med. 2014;239:1073-7.

28. Kolodka TM, Garlick JA, Taichman LB. Evidence for keratinocyte stem cells in vitro: long term engraftment and persistence of transgene expression from retrovirus-transduced keratinocytes. Proc Natl Acad Sci USA. 1998;95:4356-61.

29. Margulis A, Andriani F, Fusenig N, et al. Abrogation of Ecadherin-mediated adhesion induces tumor cell invasion in human skin-like organotypic culture. J Invest Dermatol. 2003;121: 1182-90.
30. Andriani F, Margulis A, Lin N, et al. Analysis of microenvironmental factors contributing to basement membrane assembly and normalized epidermal phenotype. $\mathbf{J}$ Invest Dermatol. 2003;120:923-31.

31. Segal N, Andriani F, Pfeiffer L, et al. The basement membrane microenvironment directs the normalization and survival of bioengineered human skin equivalents. Matrix Biol: J Int Soc Matrix Biol. 2008;27:163-70.

32. Carlson M, Faria K, Shamis Y, et al. Epidermal stem cells are preserved during commercial-scale manufacture of a bilayered, living cellular construct (Apligraf(R)). Tissue Eng Part A. 2011;17:487-93.

33. Shamis Y, Hewitt KJ, Carlson MW, et al. Fibroblasts derived from human embryonic stem cells direct development and repair of 3D human skin equivalents. Stem Cell Res \& Ther. 2011;2:10.

34. Beyer C, Schett G, Distler O, et al. Animal models of systemic sclerosis: prospects and limitations. Arthritis Rheum. 2010;62:2831-44.

35. Ruzek MC, Jha S, Ledbetter S, et al. A modified model of graftversus-host-induced systemic sclerosis (scleroderma) exhibits all major aspects of the human disease. Arthritis Rheum. 2004;50:1319-31.

36. Yamamoto T, Katayama I. Vascular changes in bleomycininduced scleroderma. Int J Rheumatol. 2011;2011:270938.

37. Yang X, Liu C, Fujino M, et al. A modified graft-versus-hostinduced model for systemic sclerosis, with pulmonary fibrosis in Rag2-deficient mice. FEBS Open Bio. 2017;7:1316-27.

38. Whitfield ML, Finlay DR, Murray JI, et al. Systemic and cell typespecific gene expression patterns in scleroderma skin. Proc Natl Acad Sci USA. 2003;100:12319-24.

39. Gardner H, Shearstone JR, Bandaru R, et al. Gene profiling of scleroderma skin reveals robust signatures of disease that are imperfectly reflected in the transcript profiles of explanted fibroblasts. Arthritis Rheum. 2006;54:1961-73.

40. Lagares D, Santos A, Grasberger PE, et al. Targeted apoptosis of myofibroblasts with the BH3 mimetic ABT-263 reverses established fibrosis. Sci Transl Med 2017;9.

41. Yang Y, Yan F, Wang L, et al. Quantification of skin stiffness in patients with systemic sclerosis using real-time shear wave elastography: a preliminary study. Clin Exp Rheumatol. 2018;36 Suppl 113:118-25.

42. Dunn AK, Wallace VP, Coleno M, et al. Influence of optical properties on two-photon fluorescence imaging in turbid samples. Appl Opt. 2000;39:1194-201.

43. Vishwanath K, Pogue B, Mycek MA. Quantitative fluorescence lifetime spectroscopy in turbid media: comparison of theoretical, experimental and computational methods. Phys Med Biol. 2002;47:3387-405.

44. Cox TR, Erler JT. Remodeling and homeostasis of the extracellular matrix: implications for fibrotic diseases and cancer. Dis Model Mech. 2011;4:165-78.

45. Bondareva A, Downey CM, Ayres F, et al. The lysyl oxidase inhibitor, beta-aminopropionitrile, diminishes the metastatic colonization potential of circulating breast cancer cells. PLoS One. 2009;4:e5620.

46. Hornstra IK, Birge S, Starcher B, et al. Lysyl oxidase is required for vascular and diaphragmatic development in mice. J Biol Chem. 2003;278:14387-93.

47. Liu X, Zhao Y, Gao J, et al. Elastic fiber homeostasis requires lysyl oxidase-like 1 protein. Nat Genet. 2004;36:178-82.

48. Martin A, Salvador F, Moreno-Bueno G, et al. Lysyl oxidase-like 2 represses Notch1 expression in the skin to promote squamous cell carcinoma progression. EMBO J. 2015;34:1090-109.

49. Zhang J, Yang R, Liu Z, et al. Loss of lysyl oxidase-like 3 causes cleft palate and spinal deformity in mice. Hum Mol Genet. $2015 ; 24: 6174-85$. 
50. Rimar D, Rosner I, Nov Y, et al. Brief report: lysyl oxidase is a potential biomarker of fibrosis in systemic sclerosis. Arthritis Rheumatol. 2014;66:726-30.

51. Chen SJ, Yuan W, Mori Y, et al. Stimulation of type I collagen transcription in human skin fibroblasts by TGF-beta: involvement of Smad 3. J Invest Dermatol. 1999;112:49-57.

52. Ghosh AK, Yuan W, Mori Y, et al. Smad-dependent stimulation of type I collagen gene expression in human skin fibroblasts by TGF-beta involves functional cooperation with p300/CBP transcriptional coactivators. Oncogene. 2000;19:3546-55.

53. Bhattacharyya S, Tamaki Z, Wang W, et al. FibronectinEDA promotes chronic cutaneous fibrosis through Toll-like receptor signaling. Sci Transl Med. 2014;6:232ra250.
54. Kohan M, Muro AF, White ES, et al. EDA-containing cellular fibronectin induces fibroblast differentiation through binding to alpha4beta7 integrin receptor and MAPK/Erk 1/2-dependent signaling. FASEB J. 2010;24:4503-12.

55. Gao AE, Sullivan KE, Black LD 3rd. Lysyl oxidase expression in cardiac fibroblasts is regulated by alpha2betal integrin interactions with the cellular microenvironment. Biochem Biophys Res Commun. 2016;475:70-75.

56. Shamis Y, Hewitt KJ, Bear SE, et al. iPSC-derived fibroblasts demonstrate augmented production and assembly of extracellular matrix proteins. Vitr Cell Dev Biol Anim. 2012;48: $112-22$. 